\title{
Optical Schrödinger Cat States in One Mode and Two Coupled-Modes Subject to Environments
}

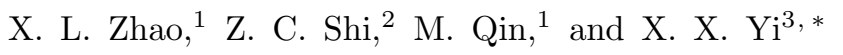 \\ ${ }^{1}$ School of Physics and Optoelectronic Technology, \\ Dalian University of Technology, Dalian 116024, China \\ ${ }^{2}$ Department of Physics, Fuzhou University, Fuzhou 350002, China \\ ${ }^{3}$ Center for Quantum Sciences and School of Physics, \\ Northeast Normal University, Changchun 130024, China
}

(Dated: June 20, 2017)

\begin{abstract}
Taking the decoherence into account, we investigate nonclassical features of the optical Schrödinger cat states in one mode and two coupled-modes systems with two-photon driving. In the one mode system, the relationship between the Schrödinger cat states and the system parameters is derived. We observe that in the presence of single-photon decay the steady states would be a mixture of Schrödinger cats. The dynamics and steady states of such a cat versus single-photon decay are examined. In the two coupled-modes cases with linear and nonlinear couplings, the dynamics of entanglement and mutual information are examined with two different initial states and single-photon decay. Compared to the linear coupling case, more complicated structure appears in the Wigner function in the nonlinear coupling case. The joint quadrature distributions are also explored. Such nonclassical states can be used not only in exploring the boundary between the classical and the quantum worlds but also in quantum metrology and quantum information processing.
\end{abstract}

\section{INTRODUCTION}

Schrödinger cat state has captured plenty of attention since it was first proposed by Schrödinger in the early days of quantum mechanics. Such kinds of macroscopic superposition states have been explored in various systems. For example, it has been studied in an ensemble of two-level atoms coupled with a cavity mode 1 and observed in superconducting quantum interference devices 2-4 . In quantum Ising model, topological defects can be put in a non-local superposition-a topological Schrödinger cat state 5. And in qubit-oscillator systems, a scheme based on conditional displacement is proposed to create Schrödinger cat states by coupling the quantum bit and harmonic oscillator [6]. Not only twocomponent Schrödinger cat states but also multicomponent Schrödinger kittens can be generated [7 7 ]. Besides, by designed double-photon processes, a Schrödinger cat state can be confined in a box [10].

In quantum optics, coherent state is a state that is very close to macroscopic state[11. Thus the superposition of two coherent states $\left|\beta_{1}\right\rangle$ and $\left|\beta_{2}\right\rangle$ (not overlapping) can be regarded as the Schrödinger cat state, i.e., $\left|\phi_{\text {cat }}\right\rangle=p_{1}\left|\beta_{1}\right\rangle+p_{2}\left|\beta_{2}\right\rangle,\left|p_{1}\right|^{2}+\left|p_{2}\right|^{2}=1$ where $p_{1}, p_{2}, \beta_{1}$ and $\beta_{2}$ are all complex. Usually the superpositions of two coherent states out of $180^{\circ}$ in phase are particularly interesting, and the superpositions of more than two coherent states would termed as Schrödinger kittens [12. Since the cat states can be composed of coherent states in quantum optics, several works have been devoted to explore the properties of these states such as squeezing, photon anti-bunching and non-Poisson distribution 13 21. The

\footnotetext{
* E-mail: yixx@nenu.edu.cn
}

Schrödinger cat states can be produced in double-photon driven-dissipative system [22], where the dissipation has negative effect on preparing these states 23. Further more, in the presence of single- and double-photon absorption and emission, exact stationary solutions for the diagonal elements of the master equation has been found 24.

In this work, we explore how the Schrödinger cat states emerge in the double-photon pumping and absorbing (decay) processes with single-photon decay in one- and two coupled-modes systems. Such cat states can be regarded as a result of competition between the double-photon generation and destruction 25. Without single-photon decay, the steady state would be a cat state with parity identical to the initial one. When the initial state is a superposition of Fock states with opposite parity, the steady state would be a weighted mixture of even and odd Schrödinger cats, and the weight of each component is determined by the initial condition. Such a relation may be revealed by constructing conserved quantities [26]. While intriguing properties emerge in the double-photon driven-dissipative process, single-photon decay is usually inevitable. Further investigation shows that the decay not only diminishes the negative interference fringes in the Wigner functions but also leads to linearly decrease of the photon number in the steady state. These however do not affect the generation of the cat state with small single-photon decay rate, and the double-photon driving can prolong the lifetime of the optical cat state.

By extending the one-mode model to two coupledmodes with linear and nonlinear couplings, the dynamical behaviors of entanglement and mutual information for two types of initial states are examined in the presence of single-photon decay. Single-photon decay not only leads to the vanishing of the negative regions in the Wigner functions. The entanglement and mutual information are 
also suppressed by the single-photon decay. Compared to the linear coupling case, entanglement and mutual information in the nonlinear coupling case are robust against the single-photon decay. And multicomponent cat states appear with sub-Planck phase interference structure in the phase space [27, 28]. Besides being used in exploring the boundary between the classical and the quantum worlds, the cat states can be used in quantum metrology [27 34 and quantum information processing 34 38.

This work is organized as follows: in Sec II, we introduce a model with double-photon driven-dissipative process for one mode system. Then the dynamics of average photon number, parity, entropy and purity with different single-photon decays are examined. Interference behaviors characterized by Wigner functions and quadrature distributions are calculated in the presence of singlephoton decay for two different initial states. In Sec III. in the cases of two modes with linear and nonlinear couplings, behavior of entanglement, mutual information for various single-photon decays with different initial states are shown. The Wigner function and joint quadrature distributions of the steady states are also explored. At last, we summarize in Sec.IV.

\section{ONE-MODE SYSTEM}

In this case, we consider the situation that the cavity mode with double-photon driving is coupled to a Markovian environment. The dynamics of this optical system $\rho$ can be described by the Lindblad master equation, $\partial_{t} \rho=i\left[\rho, \hat{H}_{a}\right]+\left(\mathcal{L}_{a}^{(1)}+\mathcal{L}_{a}^{(2)}\right) \rho(\hbar=1$ hereafter $)$. The operator $\hat{H}_{a}$ is the system Hamiltonian and $\mathcal{L}_{a}^{(1)}$ and $\mathcal{L}_{a}^{(2)}$ denote the single-photon and double-photon Lindblad dissipation super-operators. The double-photon process can be realized by Kerr effect in Josephson junctions [10]. Then we have

$$
\begin{aligned}
& \hat{H}_{a}=-\Delta_{a} \hat{a}^{\dagger} \hat{a}+\frac{U_{a}}{2} \hat{a}^{\dagger 2} \hat{a}^{2}+\frac{G_{a}}{2} \hat{a}^{2}+\frac{G_{a}^{*}}{2} \hat{a}^{\dagger 2}, \\
& \mathcal{L}_{a}^{(1)} \rho=\frac{\gamma_{a}}{2}\left(2 \hat{a} \rho \hat{a}^{\dagger}-\hat{a}^{\dagger} \hat{a} \rho-\rho \hat{a}^{\dagger} \hat{a}\right), \\
& \mathcal{L}_{a}^{(2)} \rho=\frac{\eta_{a}}{2}\left(2 \hat{a}^{2} \rho \hat{a}^{\dagger 2}-\hat{a}^{\dagger 2} \hat{a}^{2} \rho-\rho \hat{a}^{\dagger 2} \hat{a}^{2}\right),
\end{aligned}
$$

where $\Delta_{a}$ is the pump-cavity detuning $\left(\Delta_{a}=0\right.$ following) and $U_{a}$ is the photon self-interaction strength. $G_{a}$ is proportional to the degenerate double-photon parametric susceptibility. $\gamma_{a}$ denotes the strength for the single-photon dissipation and $\eta_{a}$ is the double-photon decay rate related to the cross-section for double-photon absorption and the single-photon decay destroys the coherence in one-mode case. In two modes system, the single-photon decay would influence the coupled modes through the coupling interactions. Without single-photon decay, the steady states would be semideterministic Schrödinger cat states related to the initial conditions. The optical Schrödinger cat states are generated from the competition between the double-photon parametric and destructive processes.
Define the elements of the density matrix $\langle m|\rho| n\rangle=$ $\rho_{m, n}=\sqrt{\frac{m !}{n !}} \phi_{m, n}$, where $|m\rangle$ and $|n\rangle$ are the Fock states, the density matrix elements $\phi_{m, n}$ satisfy the coupled differential equations,

$$
\begin{aligned}
\partial_{t} \phi_{m, n}= & -i\left[\frac{U_{a}}{2}(m+n-1)(m-n) \phi_{m, n}\right. \\
& +\frac{G_{a}}{2}\left((m+1)(m+2) \phi_{m+2, n}-n(n-1) \phi_{m, n-2}\right) \\
& \left.+\frac{G_{a}^{*}}{2}\left(\phi_{m-2, n}-\phi_{m, n+2}\right)\right] \\
& +\frac{\gamma_{a}}{2}\left[2(m+1) \phi_{m+1, n+1}-(m+n) \phi_{m, n}\right] \\
& +\frac{\eta_{a}}{2}\left[2(m+1)(m+2) \phi_{m+2, n+2}\right. \\
& \left.-(m(m-1)+n(n-1)) \phi_{m, n}\right]
\end{aligned}
$$

The dynamics and steady states can be obtained by solving these equations numerically in a truncated Fock state space.

\section{A. Double-photon driven-dissipative process without single-photon decay}

First, we consider the system undergoing a doublephoton driven-dissipative process but with non singlephoton decay $\left(\gamma_{a}=0\right)$. In this case, the dynamics and steady-state not only depend on the system parameters but also on the initial state since the double-photon processes link two states of the same parity. One can obtain the steady state by setting $\partial_{t} \rho=i\left[\rho, \hat{H}_{a}\right]+\left(\mathcal{L}_{a}^{(1)}+\right.$ $\left.\mathcal{L}_{a}^{(2)}\right) \rho=0$ with $\gamma_{a}=0$.

$$
\begin{aligned}
\rho_{s s}= & p_{++}|\alpha+\rangle\left\langle\alpha+\left|+p_{--}\right| \alpha-\right\rangle\langle\alpha-| \\
& +p_{+-}|\alpha+\rangle\left\langle\alpha-\left|+p_{-+}\right| \alpha-\right\rangle\langle\alpha+|,
\end{aligned}
$$

where $|\alpha \pm\rangle=\mathcal{N}_{ \pm}^{-1}(|\alpha\rangle \pm|-\alpha\rangle)$ are the even and odd Schrödinger cat states, respectively, and $\mathcal{N}_{ \pm}$are the normalization constant. We obtain the critical parameter ' $\alpha$ ' in (3) satisfying,

$$
\alpha= \pm \sqrt{\frac{G_{a}^{*}}{i \eta_{a}-U_{a}}},
$$

where $|\alpha\rangle$ and $|-\alpha\rangle$ are components for the Schrödinger cat states. Thus the desired Schrödinger cat states may be obtained by adjusting the parameters of the system. Usually large $\alpha$ is desirable since the nonclassical signatures would be more obvious for Schrödinger cat states with large average photon number.

We will use the Wigner function to characterize nonclassical states in the phase space [39], which can be reconstructed by homodyne tomography technique 40. The appearance of negative value of Wigner function is the necessary condition to indicate the appearance of nonclassical state [11. It is defined as $W(\alpha)=$ 

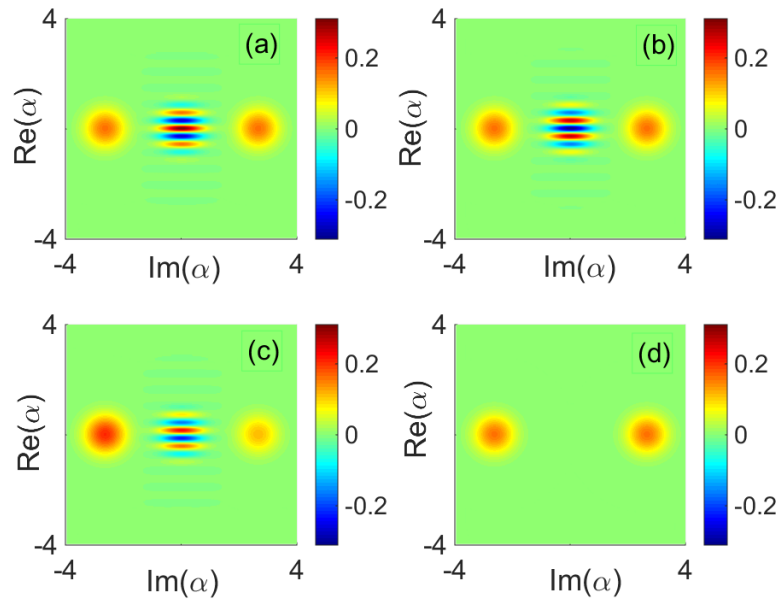

Figure 1. (a)-(d) show the Wigner functions for the steady states with the initial states, $|0\rangle\langle 0|| 1\rangle,\langle 1|, 1 / 2(|0\rangle+|1\rangle)(\langle 0|+$ $\langle 1|)$ and $1 / 2(|0\rangle\langle 0|+| 1\rangle\langle 1|)$ respectively. (a)Even Schrödinger cat state; (b) Odd Schrödinger cat state; (c) Superposition of even and odd Schrödinger cat states with different ratios; (d) Statistical mixture of even and odd Schrödinger cat states; The other parameters are $\Delta_{a}=0, U_{a} / \eta=1, G_{a} / \eta_{a}=10 e^{-i \pi / 4}$ and $\gamma_{a}=0$.

$\frac{1}{\pi} \operatorname{Tr}\left[\rho \hat{D}_{\alpha} \hat{P} \hat{D}_{\alpha}^{\dagger}\right]$, where $\hat{D}_{\alpha}=e^{\alpha \hat{a}^{\dagger}-\alpha^{*} \hat{a}}$ is the displacement operator and $\hat{P}=e^{i \pi \hat{a}^{\dagger} \hat{a}}$ is the photon parity operator. Different from the P-function, Wigner function is a quantum analog of the classical Liouville phase space probability function[11. Even though the nonclassicality of a states can be witnessed by P-function, its delicate mathematical structure makes it hard to be reconstructed by experimental data 11 . For the case of even and odd Schrödinger cat states $|\xi \pm\rangle$, the Wigner function is

$$
\begin{aligned}
W(\alpha)= & \frac{2}{\pi \mathcal{N}_{e(o)}}\left\{e^{-2|\alpha-\xi|^{2}}+e^{-2|\alpha+\xi|^{2}}\right. \\
& \left.+2 e^{-2|\alpha|^{2}}(-1)^{e(o)} \cos \left[4 \operatorname{Im}\left(\alpha^{*} \xi\right)\right]\right\} .
\end{aligned}
$$

Here $\mathcal{N}_{e(o)}=\left(1+(-1)^{e(o)} e^{-2|\xi|^{2}}\right)$ are the constants for even and odd Schrödinger cat states and the subscripts $e$ and $o$ denote even and odd Schrödinger cat states. From another point of view, the coherence of the Schrödinger cat state can also be identified by the interference fringes in quadrature distributions which can be identified by homodyne detection [13, 14. Such a distribution for a state density $\rho$ can be written as

$$
\mathcal{P}(X)=\langle X, \phi|\rho| X, \phi\rangle,
$$

where $|X, \phi\rangle$ is an eigenstate of the quadrature operator $\frac{1}{\sqrt{2}}\left(\hat{a}^{\dagger} e^{-i \phi}+\hat{a} e^{\phi}\right)$. Based on the relation $\langle X, \phi \mid n\rangle=$ $\left(2^{n} n ! \sqrt{n}\right)^{-1 / 2} H_{n}(X) e^{-X^{2} / 2} e^{-i n \phi}$, we gain the quadrature distribution of a state, here $H_{n}(X)$ are the Hermite polynomials of order $n$. Interference patterns in the
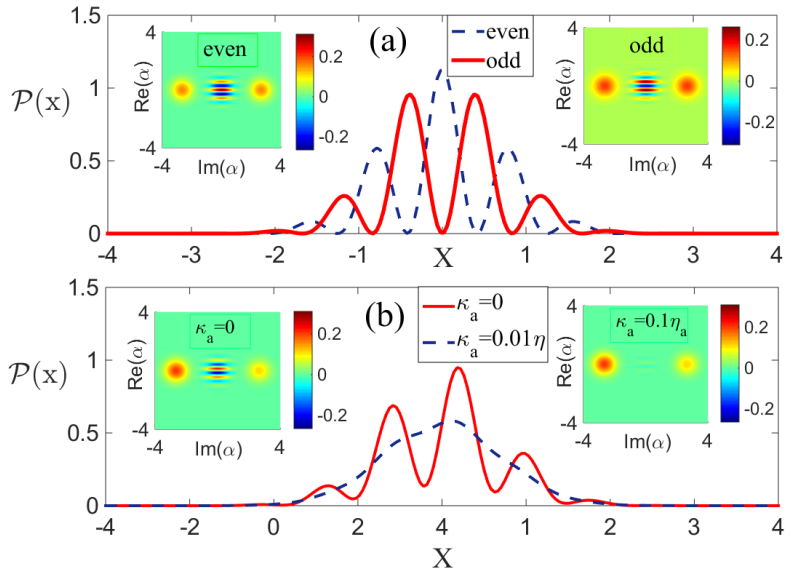

Figure 2. (a): Quadrature distributions and the corresponding Wigner functions for the steady states $\rho_{s s}$ without singlephoton decay. We have chosen the phase in quadrature operator $\phi=0$. The initial states are $|0\rangle$ (even) and $|1\rangle$ (odd) respectively. (b): The quadrature distributions and the corresponding Wigner functions for the steady states when the initial states are $1 / \sqrt{2}(|0\rangle+|1\rangle)$ for the single-photon decay rates being $\kappa_{a}=0$ and $\kappa_{a} / \eta_{a}=0.1$. The asymmetry of the quadrature distribution corresponds to that of Wigner functions. The other parameters are same to those in Fig. 1]

quadrature distributions is also a signature of nonclassical properties.

State space which is composed of even Fock states does not link to the one composed of odd Fock states by double-photon driving and decay processes. Resulting from the competition between these two double-photon processes, the initial states with deterministic even or odd parity evolve to the one with the same parity. Here they are even and odd Schrödinger cat states respectively. Whereas if the initial state is a superposition of even and odd Fock states, the steady state would be superimposed of even and odd cat states with different weights. Another case is the statistical mixture of even and odd Fock states in which the ratio of the even and odd cat states in the eigenspace of the steady state would be identical to the initial statistical one. This results from the fact that the states in even space and odd space develop separately without single-photon decay or driving process. We display the above arguments by four examples in Fig. 1.

\section{B. Double-photon driven-dissipative process in the presence of single-photon decay}

One-photon decay leads to mutual exchange of the even and odd Schrödinger cat state since $\hat{a}|\alpha \pm\rangle=\alpha|\alpha \mp\rangle$ which diminishes the interference fringes in the Wigner functions. The vanishing of the negative regions corresponds to fading away of the interference patterns in 

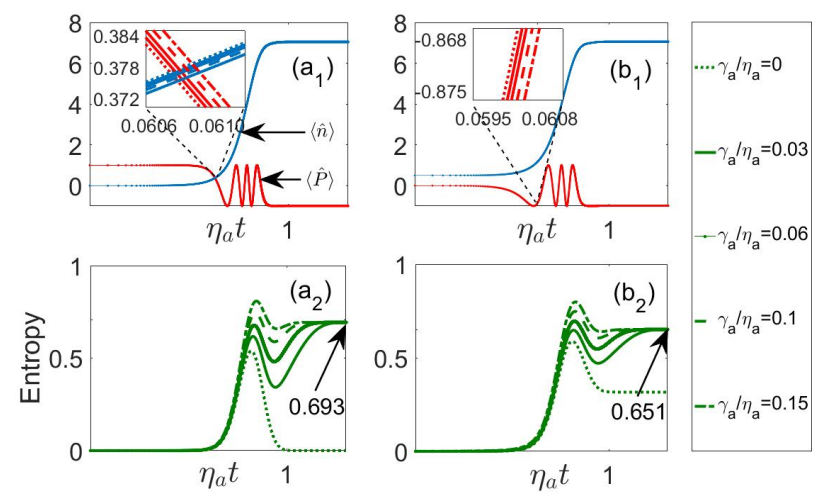

Figure 3. The evolution of the average photon number $\langle\hat{n}\rangle$ and the parity $\langle\hat{P}\rangle$ are displayed in $\left(a_{1}\right)$ and $\left(b_{1}\right)$ for different $\gamma_{a}$ and initial states. In $\left(a_{2}\right)$ and $\left(b_{2}\right)$, the transient dynamics for the entropy are displayed with the decay rates same to those in $\left(a_{1}\right)$ and $\left(b_{1}\right)$ respectively. In $\left(a_{1}\right)$ and $\left(a_{2}\right)$, the initial states are the vacuum state $|0\rangle$ while in $\left(b_{1}\right)$ and $\left(b_{2}\right)$, the initial states are $1 / \sqrt{2}(|0\rangle+|1\rangle)$. The other parameters are same to those in Fig. 1. We zoom in on $\langle\hat{n}\rangle$ and $\langle\hat{P}\rangle$ to show the weeny difference for different decay rates with the identical filate in the legend on the right.

the quadrature distributions. As an example, in Fig. 2 , the quadrature distributions are exhibited for the steady states with single-photon decay rates $\gamma_{a}=0$ and $0.1 \eta_{a}$ for two different initial states. And the interference structures in the quadrature distributions coincide with the appearance of negative stripes in the Wigner functions. With increasing of the single-photon decay strength, the negative stripe in the Wigner functions and interference fringes in the quadrature tends to fade away. And it can be seen that with the initial state $\frac{1}{\sqrt{2}}(|0\rangle+|1\rangle)$, the steady state become asymmetry in phase space and quadrature distributions.

Compared with the evolution of average photon number $\langle\hat{n}\rangle$ and parity $\langle\hat{P}\rangle$, the entropy provides more information to dissect the evolution process and it also reflects some information of the initial state. The entropy can be defined as $S(t)=-\operatorname{Tr}[\rho(t) \log (\rho(t))]$ where $\operatorname{Tr}[\bullet]$ denotes the trace of $\bullet$. We plot the transient dynamics for $\langle\hat{n}\rangle,\langle\hat{P}\rangle$ and the entropy $S(t)$ in Fig. 3 for several single-photon decay strengths with two initial states. It can be seen that while the single-photon decay is weak, the entropy would retrace after it drops from the first peak. This behavior can be interpreted as the competition between the double-photon and singlephoton processes, i.e., double-photon process dominates the entropy-decline behavior. But with time going by, as long as there is nonvanishing single-photon decay, the entropy rises and converges to an asymptotic value in long time scale. Such an asymptotic value depends on the initial condition. While the initial state is a vacuum state $|0\rangle$, the asymptotic value would be close to $\log (2)$

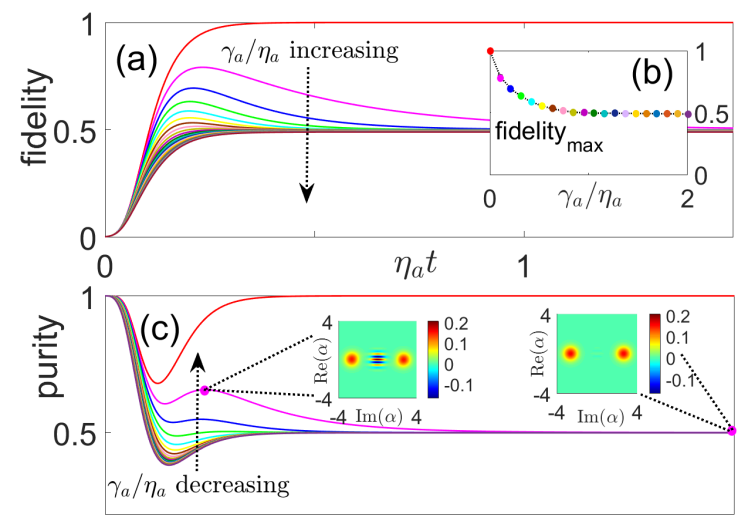

Figure 4. (a): Transient dynamics for the fidelity $\left|\left\langle\psi \mid \psi_{\text {evencat }}\right\rangle\right|^{2}$ in a range of $\gamma_{a} / \eta_{a}$ when $|0\rangle$ is set as the initial state. Panel (b) shows the maximum of the fidelity for the range of $\gamma_{a} / \eta_{a}$ during the evolution. (c): The evolution of purity defined as $\operatorname{Tr}\left[\rho^{2}\right]$ for the same range of $\gamma_{a}$ in (a). The other parameters are same to those in Fig. 1

in the presence of single-photon decay, the maximum of a two-component statical mixture in the eigenspace of the steady state. Whereas if the initial state is $(|0\rangle+|1\rangle) / \sqrt{2}$, the asymptotical value would be less than $\log (2)$. The evolution trajectory for entropy are easier to distinguish than that of $\langle\hat{P}\rangle$ and $\langle\hat{n}\rangle$ for different $\gamma \mathrm{s}$ which may be employed to reflect the strength of single-photon decay.

Even though the Schrödinger cat states vanish in the steady state in the presence of single-photon decay, it can still appear during the evolution. And the life time of a cat state can be prolonged by increasing the doublephoton dissipation compared to single-photon decay. The appearance of the Schrödinger cat state in the presence of single-photon decay is shown in Fig 4 . As shown in this figure, when $\gamma_{a} \lesssim \eta_{a}$, the fidelity can reach more than 0.5 during the evolution. The snap plots of the Wigner functions in Fig. 4 indicate the appearance of the cat states. Even though the cat states can be generated during the evolution, single-photon decay leads the fidelity approaching 0.5 in long time scale. Here the states may evolve to the statistical mixture of even and odd Schrödinger cat states with identical weights in the eigenspace. This can be confirmed by the purity defined as $\operatorname{Tr}\left[\rho^{2}\right]$. When there is single-photon decay $\left(\gamma_{a}>0\right)$, the purity converges to 0.5 in long time scale. As mentioned above, the entropy converges to $\log (2)$ implying that there are mainly two independent components(or cats) with the same weights in the steady state. These signatures suggest that the steady state is a statistical mixture of odd and even Schrödinger cat states.

Then we check the influence of single-photon decay strength to the steady state in detail. The steady state 


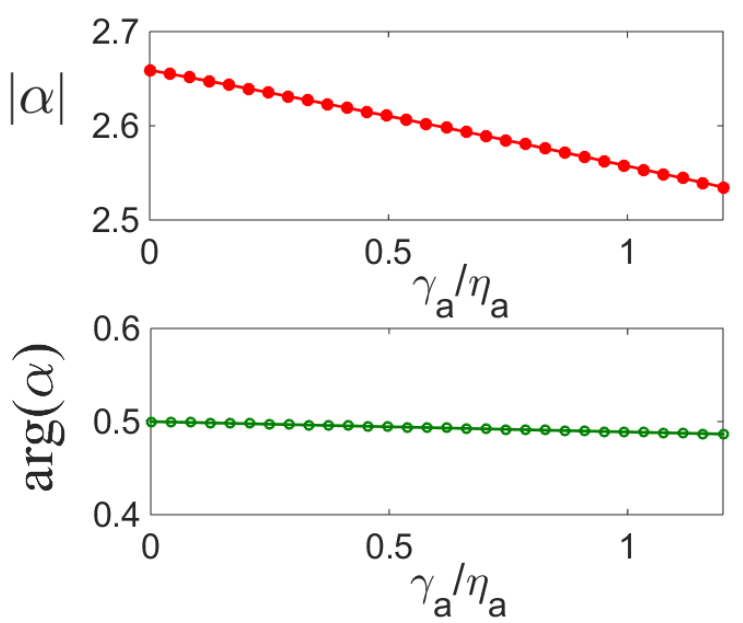

Figure 5. The influence of the single-photon decay $\gamma_{a}$ to ' $\alpha$ ' in the steady state. Here ' $\alpha$ ' denotes the 'size' of the Schrödinger cat state. The other parameters are same to those in Fig. 1

can be determined by solving the equation $\partial_{t} \rho=0$. Numerical results in Fig. 5 indicate that the modulus of $\alpha$ in the Schrödinger cat states decreases linearly as $\gamma_{a}$ increases. But the influence to the modulus of $\alpha$ is more obvious than that to the angle of $\alpha$. This means larger $\gamma_{a}$ leads to more degree of reduction of the average photon number in the steady state since $\langle \pm \alpha|\hat{n}| \pm \alpha\rangle=|\alpha|^{2}$.

\section{TWO COUPLED-MODES SYSTEM}

Based on the one-mode system discussed above, we now consider the two coupled-modes. Two cases with different kinds of couplings are considered in following, namely the case with linear coupling and the one with nonlinear coupling. These two cases can be expressed as

$$
\begin{aligned}
& \text { linear }: g_{l}\left(\hat{a} \hat{b}^{\dagger}+\hat{a}^{\dagger} \hat{b}\right) \\
& \text { nonlinear }: g_{n l}\left(\hat{a} \hat{b}^{\dagger 2}+\hat{a}^{\dagger} \hat{b}^{2}\right),
\end{aligned}
$$

where $g_{l(n l)}$ denotes the linear (nonlinear) coupling strength between the two modes. In linear coupling case, we consider two identical systems except for the phase of double-photon driving terms which influence the distributions of the Schrödinger cat states in phase space (as (4) shows). The nonlinear case is similar to the quantum description for second harmonic generation which has been considered in 41. We investigate the Schrödinger cat states in the coupled modes and correlation between the modes characterized by entanglement and mutual information. Both entanglement and mutual information reflect the correlation between the coupled

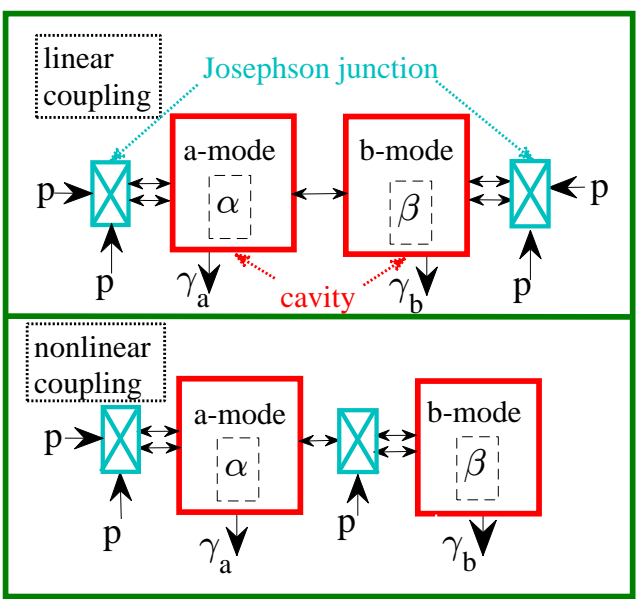

Figure 6. The sketches for the cases with linear and nonlinear couplings. The Josephson junctions provide the realization for two-photon processes by four-wave mixing process. $\alpha$ and $\beta$ in the red boxes indicate the 'size' of the coherent state components which constitute the cat states. ' $p$ 's denote the classical driving beams.

systems merely through different aspects. They play important roles in quantum information theory [42, 43. The sketches for the two coupled modes are shown in Fig. 6 .

\section{A. Influence of single-photon decay in the case with linear coupling}

First, we consider linear couplings between the two modes as shown in Fig. 6. In this case, the evolution for the total system is described by

$$
\begin{aligned}
\partial_{t} \rho= & -i\left[\hat{H}_{a}+\hat{H}_{b}, \rho\right]-i g_{l}\left[\hat{a}^{\dagger} \hat{b}+\hat{a} \hat{b}^{\dagger}, \rho\right] \\
& +\left(\mathcal{L}_{a}^{(1)}+\mathcal{L}_{a}^{(2)}+\mathcal{L}_{b}^{(1)}+\mathcal{L}_{b}^{(2)}\right) \rho .
\end{aligned}
$$

Here $\rho$ is the density matrix for the total system. $\hat{H}_{a}$ and $H_{b}$ are Hamiltonians for the two coupled modes. In this case, not only coherence behavior but also correlation between modes are worth examining. For the one mode system, the nonclassical character can be described by negative value of the Wigner functions. For the two coupled-modes, however, the entanglement and mutual information may be interesting to be examined. Since the appearance of negative eigenvalues for the partial transposed density matrix of a compound system is the necessary condition for nonseparability [43, 44, the absolute value for the summation of the negative eigenvalues of the partial transposed density matrix can be used as the entanglement indicator, i.e., the $\left|E_{-}^{T}\right|=\sum_{\lambda_{i}<0}\left|\lambda_{i}\right|$, where $\lambda_{i}$ denotes the negative eigenvalues of the partial 
transposed density matrix. Besides, quantum mutual information, or von Neumann mutual information provide us with a way to characterize the information of a variable ' $A$ ' in one system by exploring its partner ' $B$ ' in another system [45 49. It can be regarded as the quantum version of Shannon mutual information defined as $I=S_{A}+S_{B}-S_{A B}$ where $S_{A(B)}$ is the entropy for $A(B)$ mode and $S_{A B}$ is that for the compound bipartite system. For comparison, we calculate and plot the dynamics for the mutual information $I$ along with the entanglement indicator $\left|E_{-}^{T}\right|$ in Fig 7 for three examples. In the presence of single-photon decay in one of the coupled modes, the larger coupling strength leads to sharp changes in $\left|E_{-}^{T}\right|$. This can be interpreted as fast exchange of correlations between the two modes caused by larger couplings. This corresponds to single-photon decay caused destruction of nonclassical characters and the sharp destruction of the entanglement. The stronger the coupling is, the sharper the change of the mutual information. Similarly, strong couplings lead to depression of the entanglement at long time scale. Sum up, single-photon decay has negative effect on the entanglement since $\left|E_{-}^{T}\right|$ decreases sharply when the single-photon decay is nonvanishing. But the mutual information behaves robustly against this decay in the case of linear coupling.

To explore the effect of single-photon decay on the coherence, we display the Wigner functions for the coupled modes at scaled time $\eta_{a} t=2$ in Fig. 8. It can be seen that while there is single-photon decay in one of the coupled modes, the strong linear couplings lead to severe destruction to the negative regions in the Wigner functions. When the single-photon decay exists in both modes, the negative values in Wigner functions disappear at long time scales.

\section{B. Influence of single-photon decay in the case with nonlinear coupling}

In the case with nonlinear couplings, we consider a toy model in which the $a$-mode acts as the double-photon driving for another mode ( $b$-mode, i.e. the sub-converted mode) with double-photon decay. The sketch of such a model is shown in Fig. 6. The dynamics is governed by the equation,

$$
\begin{aligned}
\partial_{t} \rho= & -i\left[\hat{H}_{a}+\tilde{H}_{b}, \rho\right]-i g_{n l}\left[\hat{a}^{\dagger} \hat{b}^{2}+\hat{a} \hat{b}^{\dagger 2}, \rho\right] \\
& +\left(\mathcal{L}_{a}^{(1)}+\mathcal{L}_{a}^{(2)}+\mathcal{L}_{b}^{(1)}+\mathcal{L}_{b}^{(2)}\right) \rho, \\
\tilde{H}_{b}= & -\Delta_{b} \hat{b}^{\dagger} \hat{b}+\frac{U_{b}}{2} \hat{b}^{\dagger 2} \hat{b}^{2} .
\end{aligned}
$$

This can be seen as the cascaded double-photon down conversion scheme. In this case, the entanglement $\left|E_{-}^{T}\right|$, mutual information $I$, and nonclassical signatures behave differently with respect to those in the linear coupling case. We study the influence of single-photon decay in both modes to $\left|E_{-}^{T}\right|, I$, and coherence by three examples,

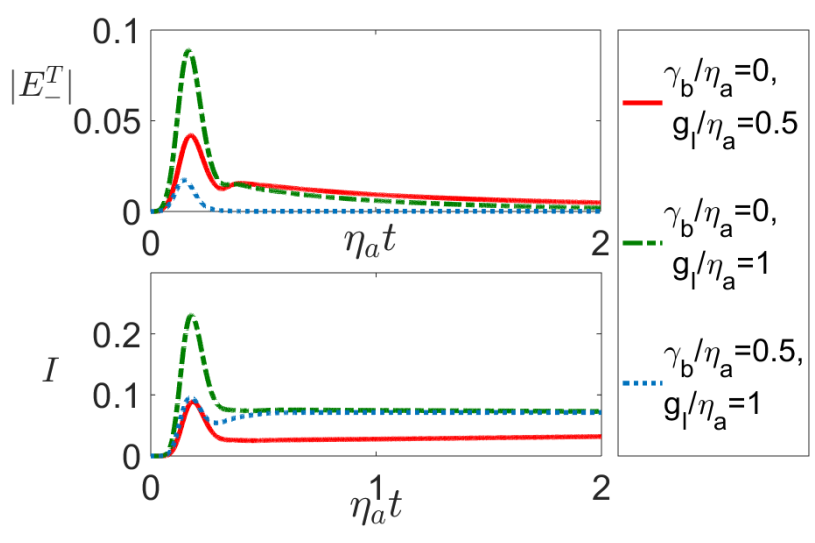

Figure 7. Transient dynamics for entanglement indicator $\left|E_{-}^{T}\right|$ and mutual information $I$ in the two modes system with linear couplings. We set $\gamma_{a} / \eta_{a}=0.5$ in this case and the other parameters are the same as those in Fig. 1. except the phase $e^{i 3 \pi / 4}$ in the double-photon driving for $b$-mode. The initial states are $|0\rangle$ for both modes.
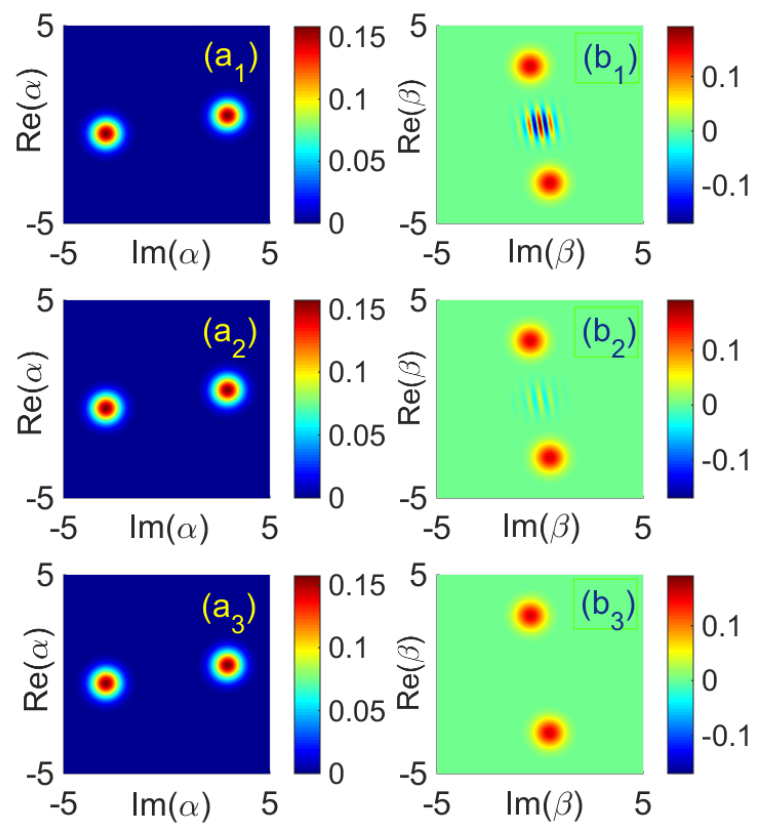

Figure 8. $\left(a_{1}\right),\left(a_{2}\right)$ and $\left(a_{3}\right)$ : The Wigner functions for $a$ mode in the case with linear couplings at time $\eta_{a} t=2$. $\left(b_{1}\right)$, $\left(b_{2}\right)$ and $\left(b_{3}\right)$ : The Wigner functions for $b$-mode corresponding to those in $\left(a_{1}\right),\left(a_{2}\right)$ and $\left(a_{3}\right)$ respectively. The other parameters in both modes are the same as those in Fig. 7.

and the results are shown in Fig. 9 and Fig. 10. When single-photon decay exists in $a$-mode, with the increasing of nonlinear coupling strength $g_{n l},\left|E_{-}^{T}\right|$ would decrease but $I$ is enhanced. When single-photon decay is added in $b$-mode $\left(\gamma_{b} \neq 0\right)$, the $\left|E_{-}^{T}\right|$ decreases obviously but the mutual information is robust against this decay. 


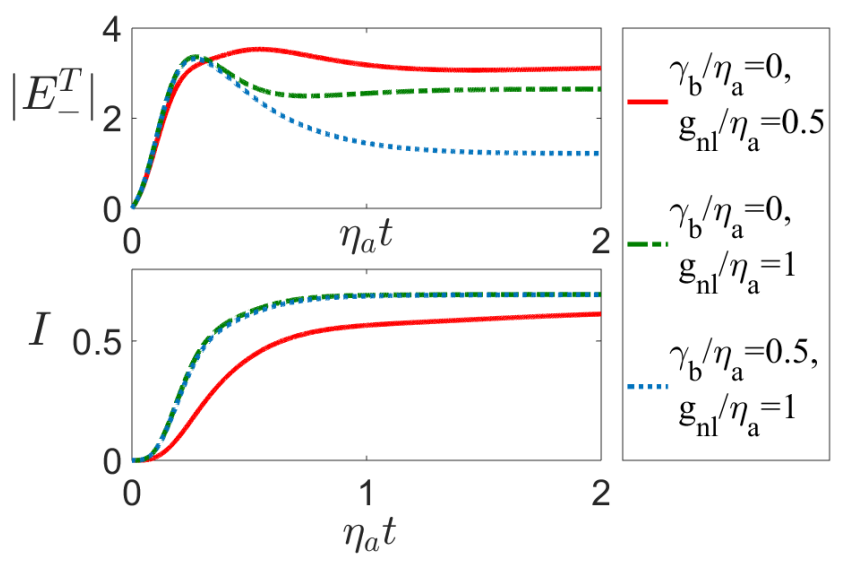

Figure 9. Transient dynamics for $\left|E_{-}^{T}\right|$ and mutual information $I$ in the case with nonlinear couplings. We set $\gamma_{a} / \eta_{a}=0.5$ in this case. The other parameters are the same as those in Fig. 11 The initial state is $|0\rangle$ for both modes.

In Fig. 10, intriguing nonclassical signature emerges in the $b$-mode when there is only double-photon decay in this mode. To reveal the intriguing patterns in both $a$ and $b$-mode, we calculate their components and show the results in Fig. 11. It can be seen that the state of $a$-mode is a superposition of even and odd Schrödinger cat states. Whereas $b$-mode is superimposed by two four-component cat-like states. The scars in the Wigner functions indicate that qubits could be encoded by these states. E.g., the qubits here can be encoded as $|\alpha\rangle_{i} \rightarrow|0\rangle_{i}$ and $|-\alpha\rangle_{i} \rightarrow|1\rangle_{i}$, where $i$ denotes the mode index and $\left| \pm \alpha_{i}\right\rangle$ are the coherent states. The Schrödinger cat states might find potential applications in quantum metrology [27 34 and may be valuable in efficient storage and communication for quantum information processing 34 38. This is due to the sensitivity of the states to the structure change in phase space, for instance, rotation and shift.

The intriguing patterns in $b$-mode may be influenced by $a$-mode though coupling. In the following, we will check the Wigner functions of $a$-mode and $b$-mode at time $\eta_{a} t=2$ by varying a set of parameters in $a$-mode in Fig. 12. We find that the double-photon driving or photon self-interaction in $a$-mode is necessary for the emergence of intriguing checkerboard patterns in $b$-mode. In turn, the pattern in $b$-mode depends on the parameters of $a$ mode. This may provide us a method to estimate system parameters by examining the coupled two modes.

\section{Schrödinger cat states in the two-mode system with two different initial states}

We calculate and show the dynamics of entanglement $\left|E_{-}^{T}\right|$ and mutual information $I$ in the two modes system for two different initial states in Fig. 13. Compared to those in Fig. 7 and Fig. 9, we find that with both linear and nonlinear couplings, $\left|E_{-}^{T}\right|$ and $I$ have been sup-
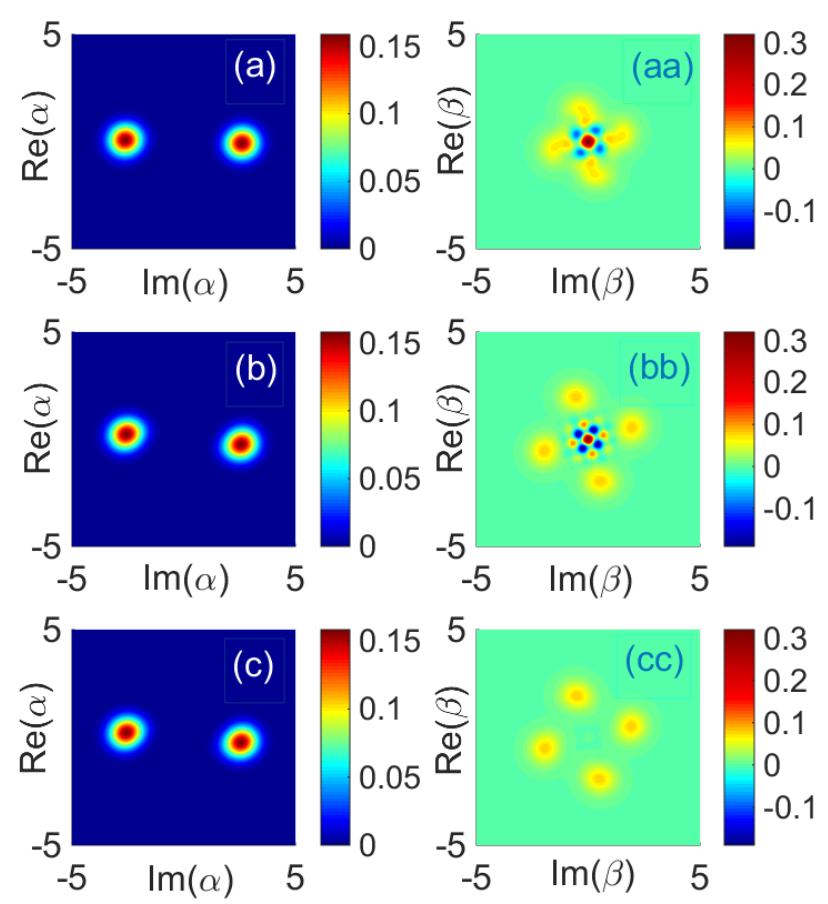

Figure 10. (a), (b) and (c): Wigner functions for $a$-mode at time $\eta_{a} t=2$ in the two modes system with nonlinear couplings.(aa), (bb) and (cc): Wigner functions for $b$-mode corresponding to (a), (b) and (c), respectively. The other parameters are the same as those in Fig. 9]

pressed. However, with nonlinear coupling, the entanglement has been suppressed more obviously compared to those in Fig. 9, while the mutual information is robust in this case.

Next we explore the change of coherence for two different initial states. Compared to Fig 8 and Fig, 10, we examine the Wigner functions at time $\eta_{a} t=2$ in Fig. 14 with the same parameters as in Fig. 13. The negative region in the Wigner functions approximately disappears in $a$-mode and small area with negative value of Wigner function left in $b$-mode in the linear coupling case. The influence on $b$-mode distribution is similar to that in the one-mode case as shown in Fig, the distribution becomes asymmetric in phase space. The distribution for $a$-mode has also become asymmetric. With nonlinear couplings, compared to those in Fig $10(b)$ and $(b b)$, the interference fringes in Fig, $14\left(a_{2}\right)$ and $\left(b_{2}\right)$ also changes obviously in $b$-mode more than that in $a$-mode. This may result from that the nonlinear coupling terms act like single-photon decay for $a$-mode. Compared to the symmetric pattern in the Wigner function (see Fig. 10, the asymmetric signatures in Fig. $14\left(b_{2}\right)$ too, resemble that in the one-mode case. 

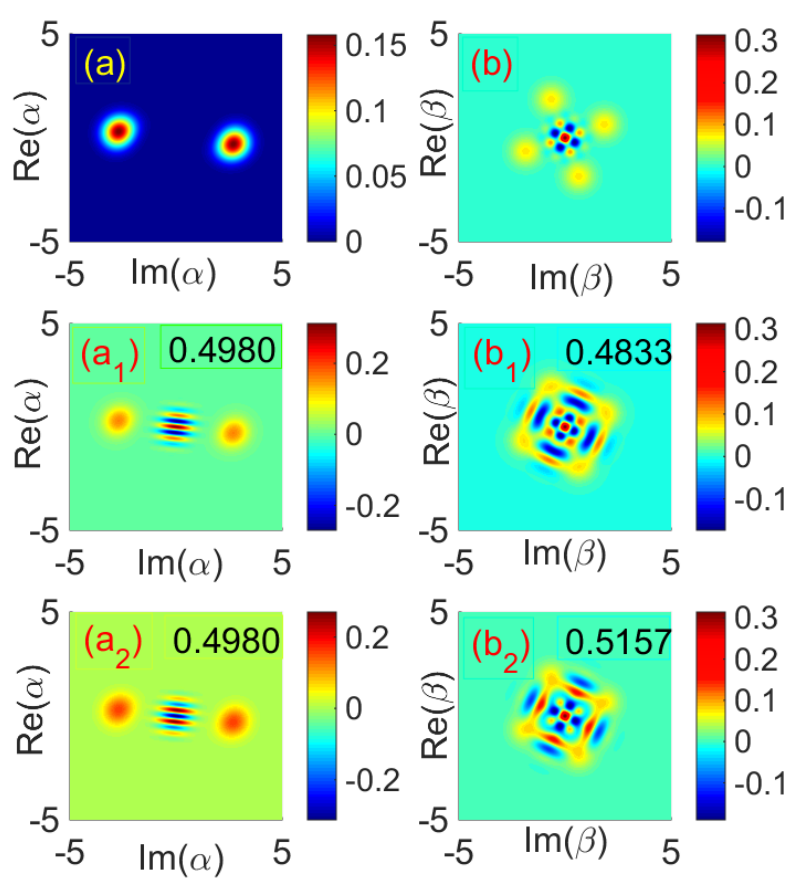

Figure 11. $(a),\left(a_{1}\right)$ and $\left(a_{2}\right)$ : The Wigner functions for $a$ mode and its two major components in its eigenspace at time $\eta_{a} t=3$ with parameters as the same as in Fig. 10(b). (b), $\left(b_{1}\right)$ and $\left(b_{2}\right)$ are those for $b$-mode with the same parameters as that in Fig. 10(bb). The decimals on the panels are the ratios for the components in the eigenspaces. The summation of major ratios are more than 0.99 .

\section{The joint quadrature distribution in the two coupled-modes cases}

In the two coupled-modes case, the joint quadrature distribution defined by $\left\langle X_{a}, X_{b}|\rho| X_{a}, X_{b}\right\rangle$ provides with the other aspects to witness the nonclassical characters of the cat states. Here $X_{a}$ and $X_{b}$ are the quadrature operators of $a$-mode and $b$-mode in (6) with $\phi=0$ and $\rho$ being the total density matrix of the coupled system. We show the Wigner functions and the corresponding joint quadrature distributions at two different times in the linear and nonlinear coupling cases in Fig, 15 and Fig 16 respectively. While there is negative regions in the Wigner functions, interference fringes also appear in the joint quadrature distributions. When the Wigner function is symmetric (asymmetric), the joint quadrature distribution is also symmetric (asymmetric). Considering the interference fringes in the quadrature distributions in one-mode case, we can see that measurement of the quadrature $X_{a}$ may project the system to a Schrödinger cat state. The joint quadrature distribution has been used to explore Einstein-Podolsky-Rosen paradox, see Refs. [50 54. The purity trends to 0.25 which hints that $a$ - and $b$-mode approaches to a statistical mix-
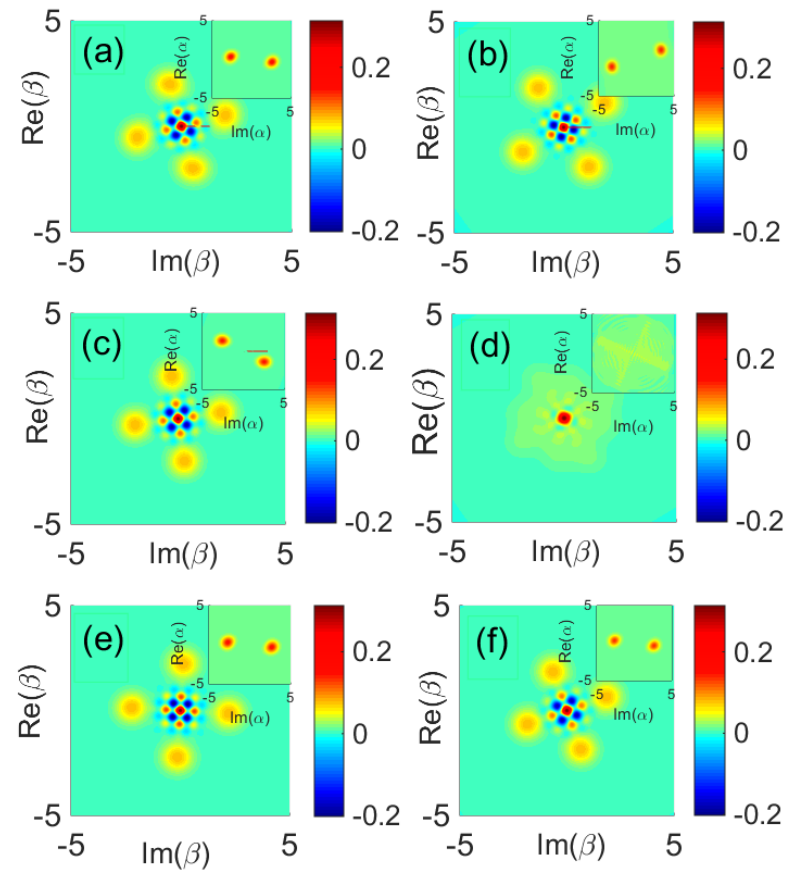

Figure 12. In (a)-(f) we set: $\gamma_{a}=(0.5,0.5,0.5,0.5,0.5,0)$, $\eta_{a}=(1,0,1,0,1,1), \quad U_{a}=(1,1,0,0,1,1), \quad U_{b}=(1,1,1,1,0,1) . \quad$ The other parameters are $G_{a}=10 e^{-i \pi / 4}, U_{b}=1, \Delta_{b}=0, \eta_{b}=1$, $\gamma_{b}=0, g_{n l}=1$. Here we choose $\left|G_{a}\right| / 10$ as the energy units since both $\eta_{a}=0$ and $U_{a}=0$ situations are examined in these examples. The insets are the Wigner functions for the corresponding $a$-modes with the same color map to $b$-modes.
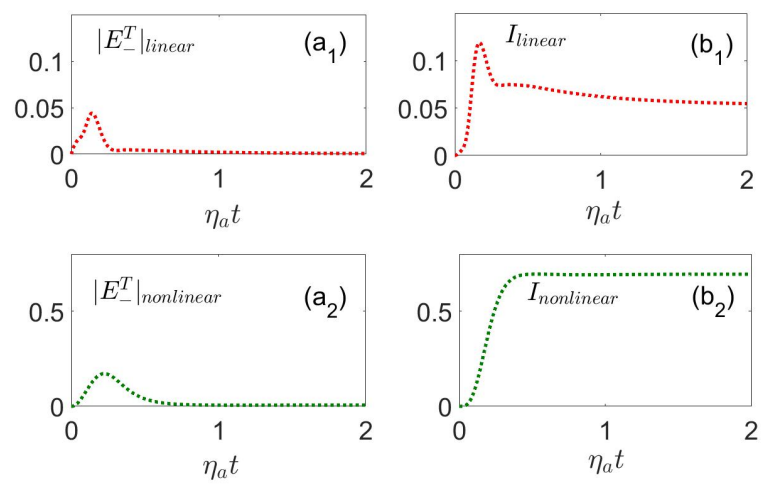

Figure 13. $\left(a_{1}\right)$ and $\left(b_{1}\right)$ show the entanglement $\left|E_{-}^{T}\right|_{\text {linear }}$ and mutual information $I_{\text {linear }}$ respectively in the case with linear couplings when $\gamma_{b} / \eta_{a}=0$ and $g_{l} / \eta_{a}=0.5$. $\left(a_{2}\right)$ and $\left(b_{2}\right)$ are those in the case with nonlinear couplings when $\gamma_{b} / \eta_{a}=0$ and $g_{n l} / \eta_{a}=0.5$. The initial state for $b$-mode are taken to be $\frac{1}{\sqrt{2}}(|0\rangle+|1\rangle)$, while it is the vacuum state for $a$-mode. The other parameters are the same as those in Fig. $8\left(a_{2}\right),\left(b_{2}\right)$ for the case with linear couplings and Fig. 10 (b), $(b b)$ for the case with nonlinear couplings respectively. 

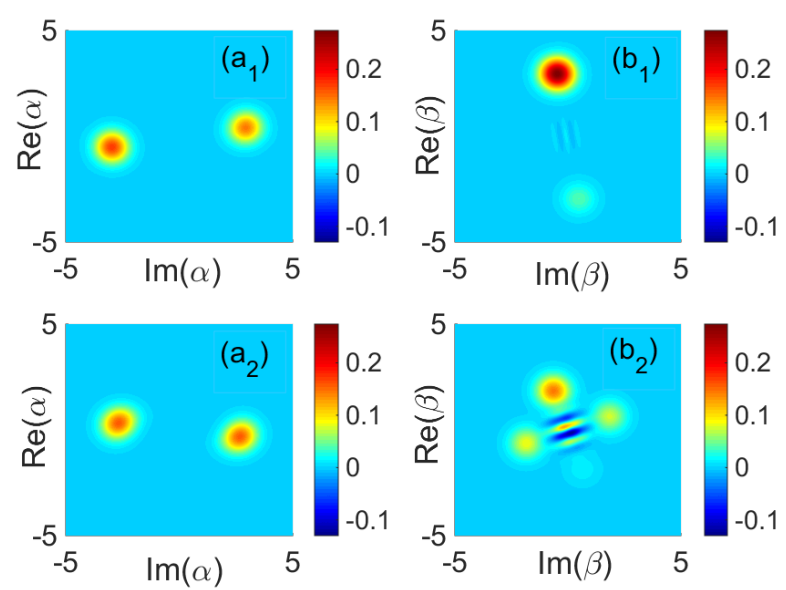

Figure 14. $\left(a_{1}\right)$ and $\left(b_{1}\right)$ show the Wigner functions for $a$ mode and $b$-mode in the linear coupling case at time $\eta_{a} t=$ 2. $\left(a_{2}\right)$ and $\left(b_{2}\right)$ are the Wigner functions in the nonlinear coupling case. The initial states and parameters in $\left(a_{1}\right),\left(a_{2}\right)$, $\left(b_{1}\right)$ and $\left(b_{2}\right)$ are the same as those in Fig. 13 In the linear coupling case, the two peaks in the Wigner function in $a$ mode are asymmetric which are more obvious than that in the nonlinear coupling case.

ture of even and odd cat states at long time scale.

\section{SUMMARY}

In summary, we have explored the Schrödinger cat states in two-photon driven dissipative systems of one mode and two cases of coupled-modes. In one mode case, the steady state is a superposition of coherent states, the weight of each component in the state is determined by the system parameters. Compared to average photon number and parity, entropy can characterize the dynamics more precisely. Single-photon decay leads to vanishing of the interference fringes in the quadrature distributions which corresponds to the disappearance of negative regions in the Wigner functions. But the Schrödinger cat states can still emerge in the presence of weak singlephoton decay. The single-photon decay slightly decreases the modulus of these coherent states in the Schrödinger cats when the system reaches the steady state. Whereas the double-photon dissipation can prolong the lifetime of the Schrödinger cats.

In the two-mode systems with both linear and nonlinear couplings, single-photon decay suppresses the entanglement at long time scales. And this deleterious effect of the single-photon decay on the entanglement is severer in the linear coupling case than that in the nonlinear coupling case. However the coupling benefits the mutual information, and intriguing nonclassical features appear in the low frequency mode in the nonlinear coupling case. But they are quite fragile against the single-photon de-
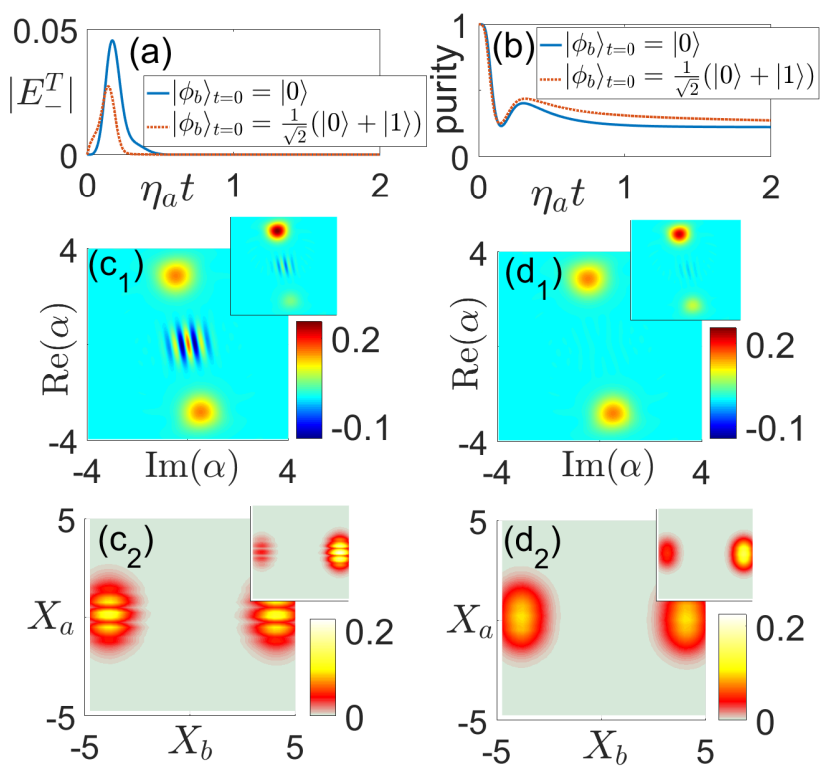

Figure 15. (a) and (b) are the evolution of the entanglement indicator $\left|E_{-}^{T}\right|$ and the purity with linear couplings for different initial states (solid curve: $\left|\phi_{b}\right\rangle=|0\rangle$; dashed curve: $\left|\phi_{b}\right\rangle=(|0\rangle+|1\rangle) / \sqrt{2}$. $\left(c_{1}\right)$ and $\left(c_{2}\right)$ are the Wigner functions for $b$-mode and the corresponding joint quadrature distributions at time $\eta_{a} t=0.4$ when $\left|\phi_{b}\right\rangle=|0\rangle . \quad\left(d_{1}\right)$ and $\left(d_{2}\right)$ are the Wigner functions for $b$-mode and the corresponding joint quadrature distributions at time $\eta_{a} t=2$. The insets are those with initial states of $b$-mode: $\left|\phi_{b}\right\rangle=(|0\rangle+|1\rangle) / \sqrt{2}$ as shown in (a) and (b) while $\left|\phi_{a}\right\rangle=|0\rangle$. We have set $\gamma_{a} / \eta_{a}=\gamma_{b} / \eta_{a}=0.1$ and $g_{l} / \eta_{a}=1$ and the other parameters are the same as those in Fig. 7

cay. Such cat states not only provide us with candidates to explore the boundary between quantum and classical world, but also can be applied in precision measurement and quantum information processing.

\section{ACKNOWLEDGMENTS}

This work is supported by the National Natural Science Foundation of China (Grant No. 11534002 and $61475033)$.
[1] G. S. Agarwal, R. R. Puri, and R. P. Singh, Atomic schrödinger cat states, Phys. Rev. A 56, 2249 (1997).
[2] C. C. Gerry, Schrödinger cat states in a Josephson junction, Phys. Rev. B 57, 7474 (1998). 

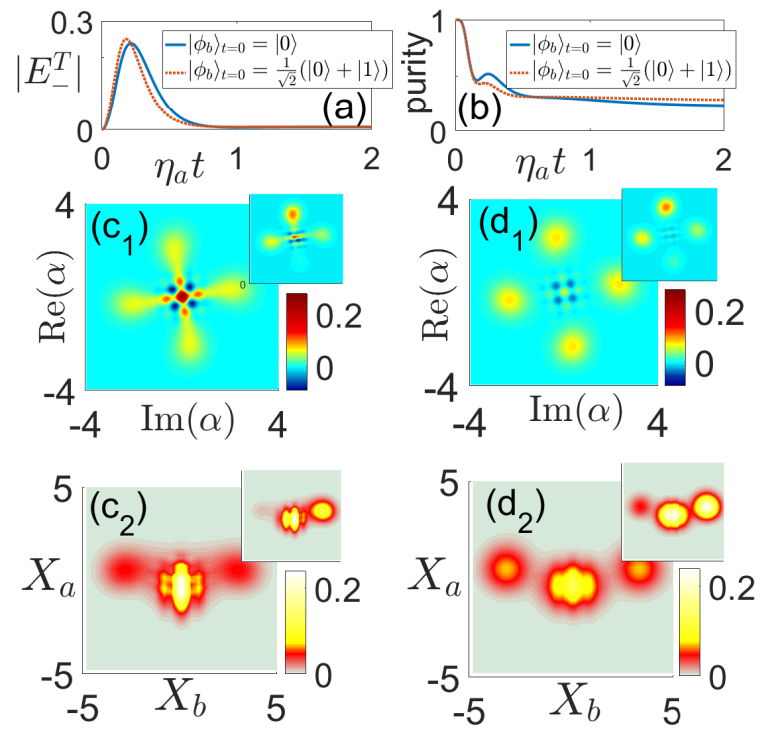

Figure 16. (a) and (b) are the evolution of $\left|E_{-}^{T}\right|$ and the purity in the case with nonlinear coupling for different initial states (solid curve: $\left|\phi_{b}\right\rangle=|0\rangle$; dashed curve: $\left|\phi_{b}\right\rangle=(|0\rangle+|1\rangle) / \sqrt{2}$. $\left(c_{1}\right)$ and $\left(c_{2}\right)$ are the Wigner functions for $b$-mode and the corresponding joint quadrature distributions at time $\eta_{a} t=0.4$ when $\left|\phi_{b}\right\rangle=|0\rangle$. $\left(d_{1}\right)$ and $\left(d_{2}\right)$ are the Wigner functions for $b$ mode and the corresponding joint quadrature distributions at time $\eta_{a} t=2$. The insets are those with initial states of $b$-mode: $\left|\phi_{b}\right\rangle=(|0\rangle+|1\rangle) / \sqrt{2}$ as shown in (a) and (b). We have set $\gamma_{a} / \eta_{a}=\gamma_{b} / \eta_{a}=0.1$ and $g_{n l} / \eta_{a}=0.5$ and the other parameters are the same as those in Fig. 9 .

[3] J. R. Friedman, V. Patel, W. Chen, S. K. Tolpygo and J. E. Lukens, Quantum superposition of distinct macroscopic states, Nature 406, 43 (2000).

[4] M. J. Everitt, T. P. Spiller, G. J. Milburn, R. D. Wilson and A. M. Zagoskin, Engineering dissipative channels for realizing Schrödinger cats in SQUIDs, Front. ICT 1,1 (2014).

[5] J. Dziarmaga, W. H. Zurek and M. Zwolak, Non-local quantum superpositions of topological defects, Nature Physics 8, 49 (2012).

[6] Jie-Qiao Liao, Jin-Feng Huang and Lin Tian, Generation of macroscopic Schrödinger-cat states in qubit-oscillator systems, Phys. Rev. A 93, 033853 (2016).

[7] S Kilin, D Mogilevtsev, The generation of multiple Schrödinger-cat states via a four-wave interaction, Physics Letters A 198, 85 (1995).

[8] D Mogilevtsev, Kilin S Ya, The generation of multicomponent entangled Schrödinger cat states via a fully quantized nondegenerate four-wave mixing process Optics Communications 132, 452 (1996).

[9] G. Kirchmair, B. Vlastakis, Z. Leghtas, S. E. Nigg, H. Paik, E. Ginossar, M. Mirrahimi, L. Frunzio, S. M. Girvin and R. J. Schoelkopf, Observation of quantum state collapse and revival due to the single-photon Kerr effect, Nature 495, 205 (2013).

[10] Z. Leghtas, S. Touzard, I. M. Pop, A. Kou, B. Vlastakis, A. Petrenko, K. M. Sliwa, A. Narla, S. Shankar, M. J. Hatridge, M. Reagor, L. Frunzio, R. J. Schoelkopf, M.
Mirrahimi, and M. H. Devoret, Confining the state of light to a quantum manifold by engineered two-photon loss, Science 347, 853 (2015).

[11] Scully, M. O., and M. S. Zubairy, 1997, Quantum Optics (Cambridge University Press, Cambridge, England).

[12] Z. Leghtas, G. Kirchmair, B. Vlastakis, M. H. Devoret, R. J. Schoelkopf, and M. Mirrahimi, Deterministic protocol for mapping a qubit to coherent state superpositions in a cavity, Phys. Rev. A 87, 042315 (2013).

[13] Yurke B. and Stoler, Generating quantum mechanical superpositions of macroscopically distinguishable states via amplitude dispersion, Phys. Rev. Lett. 57, 13 (1986).

[14] Shang Song, Carlton M. Caves, and Bernard Yurke, Phys. Rev. A 41, 5261(R) (1990).

[15] Tetsuo Ogawa, Masahito Ueda, and Nobuyuki Imoto, Generation of the Schrödinger-cat state by continuous photodetection, Phys. Rev. A 43, 6458 (1991)

[16] Buzek V., Vidiella-Barranco A. and Knight P. L., Superposition of coherent states: squeezing and dissipation, Phys. Rev. A 45, 6570 (1992).

[17] M. Reid and L. Krippner, Macroscopic quantum superposition states in nondegenerate parametric oscillation, Phys. Rev. A 47, 552 (1993)

[18] Production of Schrödinger macroscopic quantumsuperposition states in a Kerr medium, K. Tara, G. Agarwal, and S. Chaturvedi, Phys. Rev. A 47, 5024 (1993).

[19] G. Varada and G. Agarwal, Quantum-statistical properties of a particle in a double-harmonic-oscillator potential: Generation of Schrödinger-cat states, Phys. Rev. A 48, 4062 (1993).

[20] Edwin Hach III and Christopher Gerry, Generation of mixtures of Schrödinger-cat states from a competitive two-photon process, Phys. Rev. A 49, 490 (1994).

[21] C. C. Gerry and Rainer Grobe, Nonclassical properties of correlated two-mode Schrödinger cat states, Phys. Rev. A 51, 1698 (1995).

[22] C. C. Gerry, Non-classical properties of even and odd coherent states, Journal of Modern Optics. 40, 1053 (2007).

[23] D. F. Walls and G. J. Milburn, Effect of dissipation on quantum coherence, Phys. Rev. A 31, 2403 (1985).

[24] V. V. Dodonov, S. S. Mizrahi, Stationary States in Saturated Two-Photon Processes and Generation of PhaseAveraged Mixtures of Even and Odd Quantum States, Acta Phys. Slov. 48, 349 (1998).

[25] C. C. Gerry, Edwin E Hach, Generation of even and odd coherent states in a competitive double-photon process, Phys. Lett. A 174, 185 (1993).

[26] M. Mirrahimi, Z. Leghtas, V. V Albert, S. Touzard, R. J Schoelkopf, L. Jiang, and M. H Devoret, Dynamically protected cat-qubits: a new paradigm for universal quantum computation, New Journal of Physics 16, 045014 (2014).

[27] Jitesh R. Bhatt, Prasanta K. Panigrahi, and Manan Vyas, Entanglement-induced sub-Planck phase-space structures, Phys. Rev. A 78, 034101 (2008).

[28] G. S. Agarwal and P. K. Pathak, Mesoscopic superposition of states with sub-Planck structures in phase space, Phys. Rev. A 70, 053813 (2004).

[29] P. J. Leek, Storing quantum information in Schrödinger's cats, Science 342, 568 (2013).

[30] Wang C, Gao Y Y, Reinhold P, et al. A Schrödinger cat living in two boxes, Science, 352, 1087 (2016). 
[31] F. Toscano, D. A. R. Dalvit, L. Davidovich, and W. H. Zurek, Sub-Planck phase-space structures and Heisenberg-limited measurements Phys. Rev. A 73, 023803 (2006).

[32] W. J. Munro, K. Nemoto, G. J. Milburn, and S. L. Braunstein, Weak-force detection with superposed coherent states, Phys. Rev. A 66, 023819 (2002).

[33] Zurek W H. Sub-Planck structure in phase space and its relevance for quantum decoherence. Nature, 412, 712 ( 2001).

[34] Brian Vlastakis, Gerhard Kirchmair, Zaki Leghtas, Simon E. Nigg, Luigi Frunzio, S. M. Girvin, Mazyar Mirrahimi, M. H. Devoret and R. J. Schoelkopf , Deterministically Encoding Quantum Information Using 100Photon Schrödinger Cat States, Science 342, 607 (2013).

[35] A. Ourjoumtsev, R. Tualle-Brouri, J. Laurat, P. Grangier, Generating optical Schrödinger kittens for quantum information processing, Science 312, 83 (2006).

[36] A. Ourjoumtsev, H. Jeong, R. Tualle-Brouri, and P. Grangier, Generation of optical Schrödinger cats from photon number states, Nature 448, 784 (2007).

[37] H. Jeong, M. S. Kim, and Jinhyoung Lee, Quantuminformation processing for a coherent superposition state via a mixedentangled coherent channel, Phys. Rev. A 64, 052308 (2001).

[38] H. Jeong, T.C. Ralph, Schrodinger Cat States for Quantum Information Processing arXiv:quant-ph/0509137 (2005).

[39] E. Wigner, On the Quantum Correction For Thermodynamic Equilibrium, Phys. Rev. 40, 749 (1932).

[40] K. Vogel and H. Risken, Determination of quasiprobability distributions in terms of probability distributions for the rotated quadrature phase, Phys. Rev. A 40, 2847(R) (1989).

[41] P.D. Drummond , K.J. McNeil, and D.F. Walls, Nonequilibrium transition in sub/second harmonic generation. Opt. Acta 28, 211 (1981).

[42] Mark M. Wilde, Quantum Information Theory, Cambridge University Press, ( 2013).
[43] G. Vidal and R. F. Werner, Computable measure of entanglement, Phys. Rev. A 65, 032314 (2002).

[44] A. Peres, Separability criterion for density matrices, Phys. Rev. Lett. 77, 1413 (1996).

[45] C. E. Shannon and W. Weaver, The Mathematical Theory of Communication (University of Illinois Press, Champaign, 1998).

[46] T. Cover and J. Thomas, Elements of Information Theory (Wiley, New York, 1991).

[47] L. Amico, R. Fazio, A. Osterloh, and V. Vedral, Entanglement in many-body systems, Rev. Mod. Phys. 80, 517 (2008).

[48] V. Ameri, M. Eghbali-Arani, A. Mari, A. Farace, F. Kheirandish, V. Giovannetti, and R. Fazio, Mutual information as an order parameter for quantum synchronization, Physical Review A 91, 012301 (2015).

[49] V. Vedral, Classical Correlations and Entanglement in Quantum Measurements, Phys. Rev. Lett. 90. 050401 (2003).

[50] A. Einstein, B. Podolsky, and N. Rosen, Can quantummechanical description of physical reality be considered complete?, Phys. Rev. 47, 777 (1935).

[51] Y. Shen, S.M. Assad, N.?B. Grosse, X.Y. Li, M.D. Reid, and P.K. Lam, Nonlinear entanglement and its application to generating cat states, Phys. Rev. Lett. 114, 100403 (2015).

[52] M. D. Reid and P. D. Drummond, Quantum correlations of phase in nondegenerate parametric oscillation, Phys. Rev. Lett. 60, 2731 (1988).

[53] M. D. Reid, P. D. Drummond, W. P. Bowen, E. G. Cavalcanti, P. K. Lam, H. A. Bachor, U. L. Andersen, and G. Leuchs, Colloquium: The Einstein-Podolsky-Rosen paradox: From concepts to applications Rev. Mod. Phys. 81, 1727 (2009).

[54] L. Rosales-Zŕate, R. Y. Teh, S. Kiesewetter, A. Brolis, K. $\mathrm{Ng}$, and M. D. Reid, Decoherence of Einstein-PodolskyRosen steering, Journal of the Optical Society of America B 32, A 82 (2015). 